Research Article

\title{
Preparation and Evaluation of a Novel Oral Microemulsion Drug Delivery System for Enhancing the Bioavailability of Diltiazem
}

\author{
Ranajit Nath $^{1 *}$, Ratul Bhowmik ${ }^{2}$, Rajarshi Chakraborty ${ }^{1}$, Sourav Datta ${ }^{1}$, Apala Chakraborty ${ }^{1}$ \\ ${ }^{1}$ NSHM Knowledge Campus, Kolkata-Group of Institutions, Kolkata, West Bengal, India. \\ ${ }^{2}$ Department of Pharmaceutical Chemistry, SPER, Jamia Hamdard, New Delhi, India. \\ *Corresponding author's E-mail: ranajitnath465@gmail.com
}

Received: 14-07-2021; Revised: 22-09-2021; Accepted: 29-09-2021; Published on: 15-10-2021.

\section{ABSTRACT}

Diltiazem, a calcium ion cellular influx inhibitor is known for its limited and variable bioavailability. This study is intended to explore the benefits of microemulsion formulation as an oral drug delivery system for immediate release to improve the bioavailability and efficacy of Diltiazem. Methods: Oil in water microemulsion was prepared using the simple water titration method. The optimized formulation was evaluated for physicochemical parameters like viscosity, $\mathrm{pH}$, conductivity, and accelerated stability studies. In vitro release, diltiazem microemulsion was investigated.

Keywords: Target drug delivery; Drug delivery; bioavailability; microemulsion.

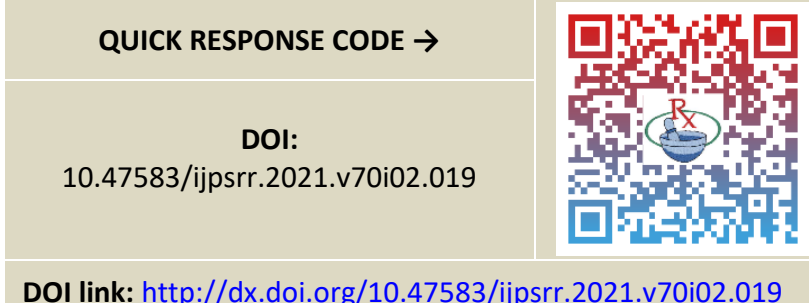

DOI link: http://dx.doi.org/10.47583/ijpsrr.2021.v70i02.019

\section{INTRODUCTION}

D iltiazem is a calcium channel antagonist and benzothiazepine derivative that is often used to alleviate symptoms and angina. Although it is well absorbed throughout the digestive tract, it has a large interindividual variability and a low absolute bioavailability $(30 \%$ to $40 \%$ ) due to considerable pre-systemic metabolism. 1-4 Cytochrome 3A4 (CYP 3A4) and intestinal P-glycoprotein (P-GP) efflux transporters are potential candidates for this action. 2-5,6 The discovery of a new drug molecule can be a time-consuming, difficult, and expensive process, but the discovery of new formulations of existing patented medication molecules opens up enormous opportunities for boosting pharmacotherapeutic properties 1,2,3. Efforts are being made to increase its bioavailability by the development of sustained-release suspensions including resin complexes and sparingly soluble salt. 7,8 These solutions lacked simplicity in terms of formulation and evaluation ${ }^{30}$. Microemulsions are a prominent drug carrier that is being researched. They are thermodynamically stable, isotropic liquid formulations with optical clarity and a simple production method of combining oil, water, and surfactant; a cosurfactant is frequently incorporated inside the formulation to increase stability by further reducing particle size ${ }^{4,5}$. Due to the advantages of microemulsions, such as their ease of preparation, thermodynamic stability, and optical clarity, an attempt was made to develop diltiazem as an oral, oil in water microemulsion formulation for immediate release. The main benefits of liquid orals are their simplicity of administration and adjustability in dose. The drug's low bioavailability was another important factor that drove the event of oral microemulsion to investigate any potential improvement in bioavailability and efficacy ${ }^{6,7}$. The inhibition of P-gp by the formulation's excipients may improve the drug's bioavailability. The study's purpose was to develop a suitable oil-in-water microemulsion for rapid release based on a water-insoluble diltiazem-free foundation to improve diltiazem bioavailability. For senior hypertension individuals, the medication's liquid oral formulation would be preferred ${ }^{8,9}$.

The notion of Micro-emulsions remained undocumented until the work of Hoar and Schulman in 1943, who described an impulsive emulsion of oil and water with the addition of a long-lasting surface-active ingredient. Schulman et al. introduced the term "micro-emulsion" in 1959 to describe a point system consisting of alcohol, oil, water, and surfactants that creates a transparent solution. Although the term "microemulsion" is no longer widely used to describe such systems, others prefer the terms "micellar emulsion" or "swollen micelles." The best definition of microemulsions is "a micro-emulsion is a system comprising oil, water, and an amphiphile that is a single optically identical and thermodynamically stable liquid solution." It is generally known that large amounts of two incompatible liquids (for example, oil and water) are frequently converted into a distinct phase (microscopically heterogeneous but macroscopically homogenous) by the addition of a suitable surface-active-agent or a surfaceactive-agent mixture 10,11,12. Micro-emulsions are an exclusive type of optically clear, thermodynamically stable, and usually low viscosity solutions. 2 Microemulsions are transparent, homogeneous, thermodynamically stable

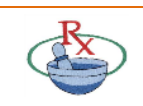


dispersions of oil and water that are normally stabilized by a surface-active-agent, ideally in conjunction with a surface-active-agent, and their general drop diameter ranges from $10-140 \mathrm{~m}$. 3 . This technique is suitable for the distribution of both water-insoluble and water-soluble medicines. Water-insoluble medicines can also be supplied via oil-in-water (o/w) microemulsions, whereas watersoluble drugs can be delivered via water-in-oil (w/o) microemulsions. micro-emulsions 4 Micro-emulsions are macroscopically similar combinations of at most a hydrophilic, a hydrophobic associate, and an amphiphilic component. Their natural philosophy stability and nanostructure square measure two necessary distinguishing features that distinguish them from conventional emulsions, which square measure thermodynamically unstable. 5 Because the area of the elements is much shorter than the wave-length of detectable light $(400-800 \mathrm{~nm})$, micro-emulsions are crystal-clear and their creation cannot be seen using an optical magnifier of the same magnification ${ }^{13,14,15}$.

\section{Microemulsion Preparation Steps}

When a surfactant and cosurfactant mixture is put into a biphasic oil-water system, a thermodynamically stable, optically transparent, or translucent, low viscosity, and isotropic mixture emerges spontaneously. The small droplet diameter of these systems contributes to their transparency $(10-200 \mathrm{~nm})$. When compared to the coarse droplets $(0.5-100 \mathrm{~m})$ of traditional or standard macroemulsions such as emollient liquids, cream, lotions, etc., such small droplets produce only a weak scattering of visible light. Structurally, micro-emulsions have normal micellar solutions, reverse micelles, cores or droplets of water or oil, and, for some systems, even bi-continuous structures could solubilize. Surfactants, lipids, permeability enhancers, micronization, salt formation, cyclodextrins, nanoparticles, and solid dispersions are among the formulation solutions used to overcome these issues. Each strategy for increasing bioavailability has advantages and disadvantages. The salts generated during salt production may revert to their original acid or basic forms, causing aggregation in the gastrointestinal tract (GIT). Particle size reduction may not be desired in instances where very fine powders have handling issues and poor wettability. One of the most critical difficulties in the field of pharmaceutics is improving drug solubility. Almost $40 \%$ of all new pharmacologically active compounds have poor water solubility, resulting in low effective concentrations in biofluids and thus poor bioavailability. Many studies have been conducted to improve the solubility and bioavailability of weakly water-soluble medicines to take them orally, hence increasing their therapeutic efficacy. The integration of the active lipophilic component into inert lipid vehicles such as oils, surfactant dispersions, selfemulsifying formulations, emulsions, and liposomes is one of the most preferred approaches ${ }^{16,17}$. Depending on their structural structure, microemulsion allows for the integration of both hydrophilic and lipophilic compounds. These aggregates have been described as reservoir systems, allowing medications to be released slowly, offering sustained effects while avoiding excessive concentrations in the circulation. Microemulsions (MEs) are isotropic, thermodynamically stable, transparent (or translucent) systems of oil, water, and surfactant, often in combination with a co-surfactant, with droplet sizes ranging from 10-100 $\mathrm{nm}$. These homogeneous systems, which can be created with a variety of surfactant concentrations and oil-to-water ratios, are all low viscosity fluids. A microemulsion as a drug delivery tool has advantageous properties such as thermodynamic stability (long shelf-life), ease of formation (zero interfacial tension and almost spontaneous formation), optical isotropy, filtration stabilizability, high surface area (high solubilization capacity), and very small droplet size. The tiny droplets also have greater membrane adhesion and deliver medication molecules in a regulated manner ${ }^{18,19}$. Over the last few decades, various colloidal forms have been evaluated as optimal pharmaceutical carriers for the oral administration of active medication. Microemulsions are isotropically transparent thermodynamically stable structures formed by combining two immiscible liquids (water and oil) to form a single phase with an appropriate surfactant or surfactant mixture ${ }^{20}$. Holland Schulman invented the term "microemulsion," which he defined as a translucent solution formed by titrating a conventional coarse emulsion with medium-chain alcohols. These are more unit stable than other types of emulsions, transparent, and usually contain a co-surfactant. Their diameters range from 10 to 140 meters. Because lipophilic pharmaceuticals are utilized as drug carriers, they require extra qualities such as drug solubilizing ability, long shelf life, increased bioavailability, preparation comfort, ultralow surface tension, and a high surface area. The goal of this study was to create pharmaceutically relevant microemulsion systems 21,22. Diltiazem, a calcium ion cellular influx inhibitor, is notorious for its low and unpredictable bioavailability. A microemulsion is an excellent choice for the oral administration of poorly water-soluble medications. Surfactant-induced alterations in GI permeability may improve medication solubilization, protect against enzymatic hydrolysis, and increase the capacity for absorption of hydrophilic, hydrophobic, and amphiphilic compounds 23,24,25. Following oral administration, it easily disperses throughout the stomach to a small droplet of the microemulsion, promoting a vast dispersion of the medicine in the GI tract. Microemulsion architectures include water in oil (w/o), oil in water (o/w), and bicontinuous systems. These aid in the absorption of the medication. If the drug's partitioning between the water and oil phases has a major impact on drug release, the microemulsion may also be able to sustain drug release. It can be created as an oil in water or a water in oil microemulsion. Oil-in-water microemulsions are regarded to be a viable technique to boost the solubility and oral bioavailability of hydrophobic goods while still maintaining their stability ${ }^{27.28}$. Hydrophobic pharmaceuticals dissolve in the dispersed phase, and the thick layer of an emulsifying agent that covers the dispersed phase 
promotes drug release. Water in oil microemulsion, on the other hand, is being investigated as a means of increasing bioavailability and maintaining the release of hydrophilic medicines across the intestinal mucosa. Diltiazem is a vasodilator and antihypertensive drug that acts by relaxing the vascular muscle and lowering blood pressure. This is related to the long-term therapeutic effects, as reducing blood pressure lowers the risk of fatal and non-fatal cardiovascular events, most notably strokes and myocardial infarctions. The purpose of this study is to investigate the advantages of microemulsion formulation as an oral drug delivery technology for quick release to improve the bioavailability and efficacy of Diltiazem. Methods: The simple water titration approach was used to create water in oil microemulsion. The optimized formulation was tested for physicochemical parameters such as $\mathrm{pH}$, zeta potential, and accelerated stability. The optimized diltiazem microemulsion's in vitro release and in vivo efficacy were evaluated ${ }^{29}$.

\section{MATERIALS AND METHODS}

\section{Materials}

Diltiazem was purchased from Balaji Drugs. Polyethylene glycol 400 (PEG 400), Olive Oil, Polysorbate 80 (Tween 80), Methanol were purchased from LOBA Chemie.

\section{Pre-formulation studies}

\section{Solubility study}

The components of the micro-emulsion were selected by solubility study using the equilibration method. A known quantity of the drug was dissolved in each of the oils, surfactants, and co-surfactants by vortexing in stoppered vials, followed by shaking at $100 \mathrm{rpm}$ for 72 hours on an orbital shaker at $25 \pm 1^{\circ} \mathrm{C}$, before centrifuging at $3000 \mathrm{rpm}$ for $15 \mathrm{~min}$. followed by filtration of supernatant liquid through $0.45 \mu \mathrm{m}$ membrane. The dissolved drug after appropriate dilution was measured for its absorbance at $237 \mathrm{~nm}$ employing a double beam UVspectrophotometer (Shimadzu UV-1700).

\section{Scanning of the drug in phosphate buffer, pH 6.8}

$10 \mathrm{mg}$ of drug was dissolved in phosphate buffer $\mathrm{pH}, 6.8$ in a $100 \mathrm{ml}$ volumetric flask, and volume was made to $100 \mathrm{ml}$ with the same solvent. This stock solution was further diluted by phosphate buffer to get the concentration of $10 \mathrm{mcg} / \mathrm{ml}$. This final solution was scanned in a UV spectrophotometer. The characteristic peak was observed at $237 \mathrm{~nm}$.

\section{Drug-excipient compatibility study}

Fourier Transform Infrared analysis (FTIR) was wont to study drug-excipients interaction by scanning the samples within the range of $400-4000 \mathrm{~cm}-1$. The pure drug was mixed with surfactant, co-surfactant, and oil, and this mixture was analyzed. The comparison was through with the FTIR spectrum of the pure drug to eliminate the likelihood of important functional groups of the drug interacting with the excipients.

\section{Formulation of Microemulsion}

Construction of Pseudoternary phase diagram based on the solubility study, expressed almond oil, tween 80 (HLB-15), and PEG 400 (HLB-11.6)were selected because of the oil phase, surfactant, and cosurfactant respectively. Surfactant: cosurfactant (Smix) were prepared at1:0, 1:1, 1:2, 1:4, 1:8, 1:10, 1:12, 2:1, 3:1, and $4: 1$ ratios, and oil was incorporated into each of those mixtures within the ratios oil: Smix $1: 1$ to $1: 9$ and the other way around by gentle shaking and vortexing. Water was added from the burette, drop in drop to the present mixture which was mixed on a magnetic stirrer. The slow and continuous stirring allows equilibration between oily and aqueous phases. The resulted mixtures ranged from a milky white, highly turbid, translucent, and transparent liquid phase. The formation of transparent, free-flowing mixtures indicated the endpoint of the titration. Other mixtures were termed unstable emulsions as they separated into two layers with time. The clear, low viscous, and transparent mixtures were termed monophasic and were kept aside for few hours for visual inspection. the varied ratios of oil, Smix, and water from the titrations that resulted during several mixtures were wont to construct a phase diagram. The three diagonals of the Triangulum represent the aqueous phase, oil phase, and Smix. The shaded area represented monophasic emulsions and thought of because of the area of existence of stable microemulsions. All titrations were repeated twice to urge reproducible results. The drug was dissolved in oil and optimized ratios of surfactant, co-surfactant, and water to urge drug-loaded microemulsions. These formulations were prepared with the oil concentration that dissolved the specified dose of diltiazem easily. The pseudo ternary phase diagrams constructed with these mixtures help to know the effect of the drug on the clear microemulsion region. The optimum formulation should have minimum Smix and maximum oil concentration so on dissolve the specified dose of the drug but with minimum gastrointestinal irritation effects of surfactants and co-surfactants.

\section{Evaluation of Microemulsion System}

pH

The $\mathrm{pH}$ of thermodynamically stable microemulsions was recorded at $25 \pm 1$ 으 $\mathrm{C}$ using (Mettler Toledo, $\mathrm{pH}$ compact 220) pH meter. The measurements were wiped out triplicate.

\section{Particle size Measurement and Zeta Potential Measurements:}

The zeta potential of the microemulsion was determined by dynamic light scattering, 


\section{In-vitro Release Kinetics}

The drug permeability was checked to employ a Franz diffusion cell fitted with a 0.45 -micron cellulose membrane pre-hydrated in water at 25 으 for twentyfour hours. The receptor compartment was crammed with $6.8 \mathrm{pH}$ phosphate buffer and therefore the donor compartment was charged with $10 \mathrm{mg}$ of pure drug or 5 $\mathrm{ml}$ of the microemulsions. The diffusion medium was continuously stirred at $100 \mathrm{rpm}$ throughout the experiment employing a magnetic stirrer (Remi $2 \mathrm{MLH}$ ). At a regular interval of $1 \mathrm{~h}, 2 \mathrm{ml}$ of the sample was withdrawn from the receptor compartment for $8 \mathrm{~h}$ and immediately replaced with an equal volume of fresh buffer. These samples were diluted with diffusion medium and therefore the absorbance was measured spectrophotometrically at $237 \quad \mathrm{~nm}$. the share cumulative drug release decided for the pure drug also because the formulated microemulsions and data were fitted to Zero order, First order, Higuchi root model, and Korsmeyer-Peppas model as per equations 1,2,3 and 4 respectively. Qt represents the cumulative percentage of the drug released in time $t(\%), Q 0$ the share of the drug in the formulation at 0th hour, k0 represents the zero-order rate constant, the primary order $\mathrm{k} 1$ rate constant $(\mathrm{h}-1) \mathrm{kH}$ Higuchi rate constant (\% $-1 / 2)$, where $r$ is the radius of the spherical particle in $\mathrm{cm}, \mathrm{n}$ is Fickian diffusion and $2 \mathrm{n}$ is case II transport which is release exponents.; $a$ and $b$ and represent structural and geometric properties of the microparticles.

\section{Accelerated stability}

The accelerated stability study was conducted at $25 \circ \mathrm{C} \pm$ $2{ }^{\circ} \mathrm{C}$ and $10^{\circ} \mathrm{C} \pm 2{ }^{\circ} \mathrm{C}$ in stability chambers (Thermo labs) as per $\mathrm{ICH}$ guidelines. Approximately $5 \mathrm{ml}$ of the formulation was stored in a tightly screw-capped bottle. During the 2 months duration of the study, samples were withdrawn at one, two months periods to see physical appearance, phase separation at accelerated gravity, and drug content.

\section{Dilution test}

The microemulsions were further taken for the dilution test for visual assessment and assessed either clear, less clear, or milky appearance. Dilution tests are supported the very fact the emulsion is miscible with the liquid that forms its continuous phase. The microemulsion resulting from dilution with continuous phase shouldn't show any separation a minimum of after one hour of storage.

\section{Centrifugation}

To eliminate metastable systems, the chosen drugloaded microemulsions were centrifuged (Research Centrifuge, R-24, Remi Instruments Limited, Mumbai) at $4000 \mathrm{rpm}$ for $4 \mathrm{~h}$.

\section{Visual inspection}

Visual inspection was made after each addition of water to the oil and surfactant or surfactant and co-surfactant mixture. The samples were identified as microemulsion, emulsion, or gel formation by visual observation.

\section{Freeze thaw cycle}

Three freeze-thaw cycles between $-10^{\circ} \mathrm{C}$ and $+25{ }^{\circ} \mathrm{C}$ with storage at each temperature for not but $48 \mathrm{~h}$ was finished the formulations. Those formulations, which passed these thermodynamic stress tests, were further taken for the dispersibility test for assessing the efficiency of self-emulsification. The formulations were observed visually for any phase separation or color change.

\section{RESULTS \&DISCUSSION}

\section{Criteria for component selection}

The microemulsion formulation consists of 1 or more surfactants together with cosurfactants and therefore the drug dissolved in oil. Hence, studying the solubility of drug altogether components of microemulsion system is a crucial parameter, particularly in oil. The drug diltiazem-free base, used for the event of the microemulsion is water-insoluble and therefore the amount of oil utilized in the system should be sufficient to solubilize the drug and maintain it in solubilized form. The surfactants and co-surfactants tween 80 and PEG 400 showed maximum solubility with $117.6 \mathrm{mg} / \mathrm{ml}$ and $105.6 \mathrm{mg} / \mathrm{ml}$.

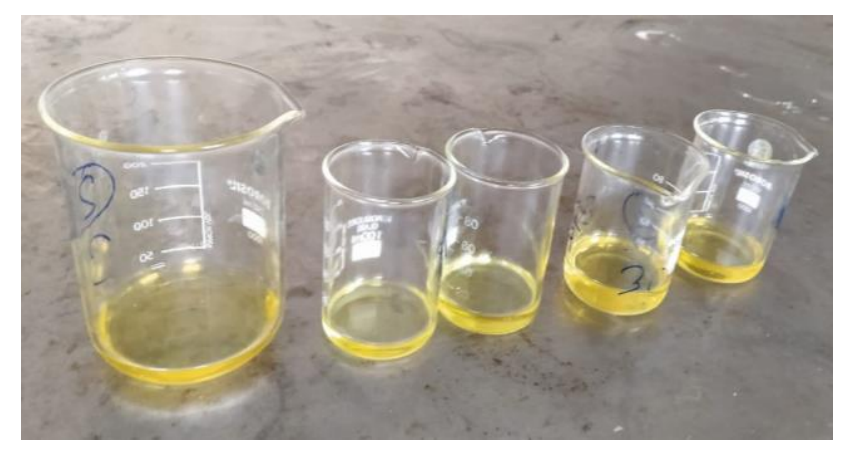

Figure1: Photo of the Formulations

\section{Drug excipient compatibility studies}

These are among the important pre-formulation studies, where any possible interaction of diltiazem with other components within the formulation affecting its pharmacological activity is studied. The FTIR spectrum indicated that none of the components interfere with the functional group of the drug. So it had been concluded that there have been no major interactions between the drug and excipients utilized in the study. 
Table 1: FTIRs of drug-excipient compatibility studies

\begin{tabular}{|c|c|c|}
\hline Component & Range & Functional group \\
\hline Drug & 500,1200 & C-H bonding, C-O stretching \\
\hline Drug+Surfactant & $500,1098,2920$ & C-H bonding, C-O stretching, C-H stretching \\
\hline Co-surfactant + Drug & 1086,2894 & C-N stretching, C-H stretching \\
\hline Co-surfactant+Surfactant+Drug+Oil & 1094,2918 & C-N stretching, C-H stretching \\
\hline Co-surfactant+Surfactant & 1087,2983 & C-N stretching, C-H stretching \\
\hline Drug+Oil & 2919,1459 & C-N stretching, C-H stretching, C-C stretching \\
\hline
\end{tabular}

\section{Pseudo ternary phase diagrams}

The conventional aqueous titration method (low energy emulsification technique) was wont to construct pseudo ternary phase diagrams for emulsions that were monophasic, clear, free-flowing, less viscous. expressed almond oil as oil phase, Tween 80 and PEG 400 as Smix phase, and purified water as aqueous phase were selected for the development of pseudo ternary phase diagram. The shaded area indicates oil in the water region and therefore the other region within the diagram indicates turbid and traditional emulsions (Figure 2). it's clear that when surfactant and cosurfactant (Smix) concentration is low the emulsion formed is biphasic and unstable. Such emulsions are turbid and milky white. because the Smix ratio increases, the clear, transparent microemulsions are formed which are stable and there's no phase separation. Another important factor is that the concentration of oil within the microemulsion system which is required to solubilize the utmost quantity of the drug. because the oil concentration increases, phase separation occurs. to stop phase separation, the Smix ratio has got to be increased thus leading to a microemulsion system, which can cause gastric irritation. Such formulations are highly viscous and not free-flowing. Hence the pseudo ternary phase diagram gives the relevant information on the optimum ratio of oil, surfactant, and cosurfactant that has got to be used for the preparation of a thermodynamically stable microemulsion system. to see the usefulness of the formulated microemulsion as a suitable drug delivery system for Diltiazem, the drug-like one dose is incorporated into the oil phase and similar phase diagrams were constructed. The phase behavior of the drug-loaded system didn't change. The monophasic, clear, and transparent formulations were further evaluated for thermodynamic stability to optimize the simplest formulations presented.

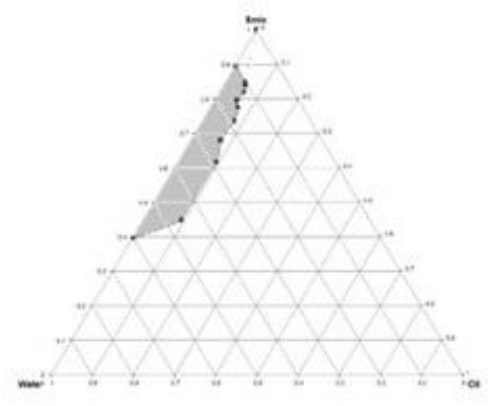

Figure 2: Pseudo ternary phase diagrams

pH

The $\mathrm{pH}$ recorded for the microemulsion containing the drug was $6.54 \pm 0.08$.

Particle size Measurement and Zeta Potential Measurements:

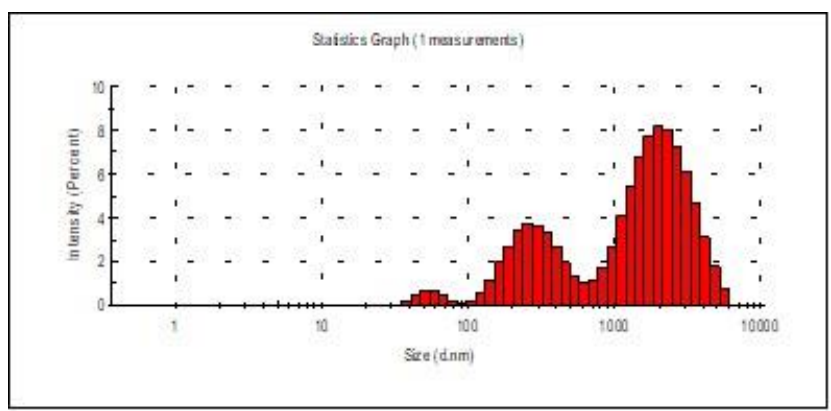

Figure 3: Particle size Measurement and Zeta Potential Measurements

\section{In-vitro drug release kinetics}

The formulations that passed the thermodynamic stability test, were further evaluated for drug release kinetic behavior. the utmost parametric statistic (R2 value) among all the models, concluded the simplest fitting model, obeyed by the formulation for drug release Kinetics.

Table 2: $R^{2}$ values

\begin{tabular}{|c|c|}
\hline Model Name & R2 value \\
\hline First Order & 0.981 \\
\hline Zero Order & 0.9811 \\
\hline Higuchi & 0.9066 \\
\hline Korsmeyer & 0.9573 \\
\hline Hixson & 0.6684 \\
\hline
\end{tabular}




\section{Accelerated stability study}

The physical examination of formulations subjected to accelerated stability study, at end of two months showed that the microemulsion can remain stable over an extended period. The microemulsion remained clear and didn't show any creaming or cracking, thus indicating honest stability.

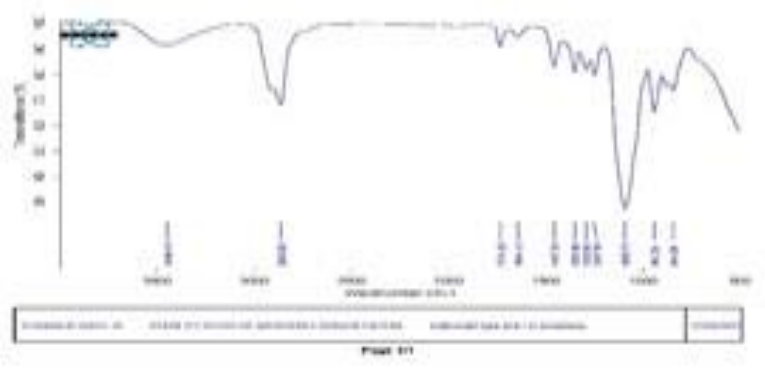

Figure 4: Accelerated stability study

\section{Physical Inspection}

Table : Physical Inspection

\begin{tabular}{|c|c|}
\hline Parameter & Condition after $\mathbf{1}$ month \\
\hline Clarity & No turbidity \\
\hline Phase separation & No phase separation \\
\hline
\end{tabular}

\section{CONCLUSION}

A microemulsion formulation containing diltiazem was successfully prepared, which exhibited optimum thermodynamic stability with appreciably higher in vitro release and superior bioavailability compared to the marketed tablet formulation. The efficacy evaluation suggested the superiority of the diltiazem microemulsion formulation over the normally marketed formulation. This study provides insight into the potential advantages of diltiazem formulated as microemulsions over the prevailing oral formulations.A pseudo-ternary phase diagram for the three microemulsion systems was delineated. Conductivity studies depicted the structural changes from w/o to o/w via the bicontinuous phase. Particle size, zeta potential studies were performed on selected formulations.

\section{REFERENCES}

1. Kwatra B, Khatun A, Bhowmik R, Rehman S. In silicomodelling of phytochemicals in septic arthritis. Pharma Innov. 2021; 10(3): 14-21.

2. Roy R, Bhowmik R, Seth S, Bhattacharyya S, Sengupta S. Computational Repurposing Model of Curcumin as a Drug. Int J Pharm Sci Rev Res. 2021; 68(1): 165-71.

3. Paul J, Debnath P, Nath R, Bhowmik R, Farheen S. Computer Aided Drug Screening for Lung Infection. Int J Pharm Sci Rev Res. 2021; 68(1): 172-6.

4. Kwatra B, Roy R, Bhowmik R, Sengupta S. DRUG REPURPOSING : IN SILICO MODELING OF COVID-19. Res J Life Sci Bioinformatics, Pharm Chem Sci. 2021; 7(2): 19-40.

5. Xie L, Wei H, Kou L, Ren L, Zhou J. Antibiotic drug release behavior of poly (vinyl alcohol)/sodium alginate hydrogels. Materwiss Werksttech. 2020; 51(7): 850-5.

6. Rodríguez Padial L, Barón-Esquivias G, Hernández Madrid A, Marzal Martín D, Pallarés-Carratalá V, de la Sierra A. Clinical Experience with Diltiazem in the Treatment of Cardiovascular Diseases. Cardiol Ther. 2016; 5(1): 75-82.

7. Zarmpi P, Flanagan T, Meehan E, Mann J, Fotaki N. Impact of Magnesium Stearate Presence and Variability on Drug Apparent Solubility Based on Drug Physicochemical Properties. AAPS J. 2020; 22(4): 1-18.

8. Kamath H, Sivakumar A. Enhanced systemic exposure and efficacy of Diltiazem from Novel Microemulsion Formulation: Characterization, in vitro release, in vivo pharmacokinetic and efficacy evaluation. J Young Pharm. 2017; 9(3): 422-8.

9. Article R. PREPARATION AND IN-VITRO EVALUATION OF MICRO EMULSION OF ANTI-HYPERTENSIVE DRUG: VALSARTAN. Int J Pharm Sci Res. 2012; 3(8): 3493-501.

10. Taufik I, Astuti DI, Purwasena IA, Rodiana WN. Effect of lemongrass (Cymbopogon citratus) essential oil on biofilmbiocorrossion in formation water environment. Asian J Sci Res. 2018; 11(3): 329-36.

11. Gupta MK, Swarnkar SK. Preformulation Studies of Diltiazem Hydrochloride. J Drug Deliv Ther. 2018; 8(1): 649.

12. Lavanya N, Aparna C, Umamahesh B. Formulation and evaluation of glipizide microemulsion. Int J Pharm Pharm Sci. 2016; 8(8): 171-6.

13. Chaudhari PM, Kuchekar MA. Development and evaluation of nanoemulsion as a carrier for topical delivery system by box-behnken design. Asian J Pharm Clin Res. 2018; 11(8): 286-93.

14. Chaudhary A, Gaur, PK, Barman M, Mishra R, Singh M. a Review on Microemulsion a Promising Optimising Technique Used As a Novel Drug Delivery System. Int Res J Pharm. 2018; 9(7): 47-52.

15. Costa AP, Xu X, Burgess DJ. Freeze-anneal-thaw cycling of unilamellar liposomes: Effect on encapsulation efficiency. Pharm Res. 2014; 31(1): 97-103.

16. de Andrade DF, Zuglianello C, Pohlmann AR, Guterres SS, Beck RCR. Assessing the In Vitro Drug Release from LipidCore Nanocapsules: a New Strategy Combining Dialysis Sac and a Continuous-Flow System. AAPS PharmSciTech. 2015; 16(6): 1409-17.

17. Saha S, Ramesh R. Design of prolonged release nanoparticulate formulation for diltiazem hydrochloride by central composite design. Der Pharm Lett. 2016; 8(17): 95105.

18. Siriah TM, Puranik PK, Trivedi HR. Experimental design approach for development of novel microemulsion system and immediate release self microemulsifying tablet of nebivolol HCl. Brazilian J Pharm Sci. 2020; 56:1-20.

19. Pharmacia Lettre $D$, Singh $M K$, Chandel V, Gupta V, Ramteke S. Formulation development and characterization of microemulsion for topical delivery of Glipizide. Der Pharm Lett [Internet]. 2010; 2(3): 33-42. Available from: www.scholarsresearchlibrary.com 
20. Kamaraj N, Rajaguru PY, Issac P kumar, Sundaresan S. Fabrication, characterization, in vitro drug release and glucose uptake activity of 14-deoxy, 11, 12didehydroandrographolide loaded polycaprolactone nanoparticles. Asian J Pharm Sci [Internet]. 2017; 12(4): 353-62. Available from: http://dx.doi.org/10.1016/j.ajps.2017.02.003

21. Khadka P, Ro J, Kim H, Kim I, Kim JT, Kim H, et al. Pharmaceutical particle technologies: An approach to improve drug solubility, dissolution and bioavailability. Asian J Pharm Sci [Internet]. 2014; 9(6): 304-16. Available from: http://dx.doi.org/10.1016/j.ajps.2014.05.005

22. Lin Z, Zhou D, Hoag S, Qiu Y. Influence of Drug Properties and Formulation on In Vitro Drug Release and Biowaiver Regulation of Oral Extended Release Dosage Forms. AAPS J. 2016; 18(2):3 33-45.

23. Lu Y, Wu K, Li L, He Y, Cui L, Liang N, et al. Characterization and evaluation of an oral microemulsion containing the antitumor diterpenoid compound ent-11alpha-hydroxy15- oxo-kaur-16-en-19-oic-acid. Int J Nanomedicine. 2013; 8: 1879-86.

24. Padial LR, Barón-Esquivias G, Madrid AH, Martín DM, Pallarés-Carratalá $V$, de la Sierra A. Erratum to: Clinical Experience with Diltiazem in the Treatment of Cardiovascular Diseases (Cardiology and Therapy, (2016), 5, 1, (75-82), 10.1007/s40119-016-0059-1). Cardiol Ther. 2016;5(1):83-4.
25. Savjani KT, Gajjar AK, Savjani JK. Drug Solubility: Importance and Enhancement Techniques. ISRN Pharm. 2012; 2012(100 mL):1-10.

26. Wallenwein CM, Nova MV, Janas C, Jablonka L, Gao GF, Thurn $\mathrm{M}$, et al. A dialysis-based in vitro drug release assay to study dynamics of the drug-protein transfer of temoporfin liposomes. Eur J Pharm Biopharm [Internet]. 2019; 143(May): 44-50. Available from: https://doi.org/10.1016/j.ejpb.2019.08.010

27. Kwatra B, Hussain MS, Bhowmik R, Manoharan S. Reviewing Therapeutic and Immuno-Pathological Applications of Vitamins and Carotenoids. Int J Sci Res Sci Technol. 2020; 7(4): 287-313.

28. Bhowmik R, Nath R, Roy R. Screening of Zinc Database against Streptococcal Cysteine Protease Enzyme for Identification of Novel Group A Streptococcus Inhibitors. J Pharm Res Int. 2021; 33(43A): 401-12.

29. Bhowmik R, Roy S, Sengupta S, Sharma S. Biocomputational and Pharmacological Analysis of Phytochemicals From Zingiber Officinale ( Ginger ), Allium Sativum ( Garlic ), and Murrayakoenigii ( Curry .... Zingiber Officinale (Ginger ), Allium Sativum ( Garlic ), and Murrayakoenigii ( Curry Leaf). Int J Appl Pharm. 2021; 13(5): 1-7.

30. Sengupta $S$, Bhowmik R, Acharjee $S$, Sen $S$. In-Silico Modelling of 1- 3- [ 3- ( Substituted Phenyl ) Prop- 2-Enoyl) Phenyl Thiourea Against Anti-Inflammatory Drug Targets. Biosci Biotechnol Res Asia. 2021; 18(2): 413-21.

Source of Support: The author(s) received no financial support for the research, authorship, and/or publication of this article.

Conflict of Interest: The author(s) declared no potential conflicts of interest with respect to the research, authorship, and/or publication of this article.

For any question relates to this article, please reach us at: editor@globalresearchonline.net New manuscripts for publication can be submitted at: submit@globalresearchonline.net and submit_ijpsrr@rediffmail.com 